\title{
Influence of Adhesive Systems on Bond Strength between Fiber Posts and Composite Resin Cores in a Pull-out Test Design
}

\author{
Karl-Thomas WRBAS $^{1}$, Jörg Fabian SCHIRRMEISTER ${ }^{1}$, Markus Jörg ALTENBURGER ${ }^{1}$, Anastasia \\ AGRAFIOTI $^{1}$ and Andrej Michael KIELBASSA ${ }^{2}$ \\ ${ }^{1}$ Department of Operative Dentistry and Periodontology, University School and Dental Hospital, Albert-Ludwigs-University \\ of Freiburg, Freiburg, Germany \\ ${ }^{2}$ Department of Operative Dentistry and Periodontology, University School of Dental Medicine, Campus Benjamin Franklin, \\ Charité-Universitätsmedizin Berlin, Berlin, Germany \\ Corresponding author, Karl-Thomas WRBAS; E-mail: thomas.wrbas@uniklinkik-freiburg.de
}

Received October 5, 2006 /Accepted January 25, 2007

The aim of this study was to evaluate the effects of post surface conditioning with adhesive systems on tensile bond strength between two composite resin core systems and FRP posts (ER DentinPost). Forty-eight posts were trimmed at the coronal part, and the upper part of $3 \mathrm{~mm}$ was covered with a standardized composite resin core build-up. Twenty-four posts were treated with the respective adhesive systems. Four groups were formed: G1 - ClearfilCore; G2 - Clearfil New Bond + ClearfilCore; G3 - MultiCore Flow; and G4 - AdheSE + MultiCore Flow. Mean (SD) bond strengths in MPa were 7.53 (0.89) for ClearfilCore and 8.08 (0.93) for New Bond + ClearfilCore; 5.80 (0.39) for MultiCore Flow and 5.92 (0.43) for AdheSE + MultiCore Flow. ClearfilCore achieved significantly higher bond strengths than MultiCore Flow (two-way ANOVA; $\mathrm{p}<0.0001)$. In conclusion, composite resin core materials exerted a significant influence on tensile bond strength, while adhesive systems did not significantly affect the results.

Keywords: Fiber post, Surface conditioning, Adhesive systems

\section{INTRODUCTION}

Endodontically treated teeth are often severely damaged with little remaining coronal tooth structure. As such, they require a post to retain the core build-up. A review of the pertinent literature on this subject demonstrated that cast metal posts and cores have been used in these situations to ensure retention for prosthetic rehabilitation ${ }^{1)}$. Current techniques and recommendations for treatment planning after successful root canal filling include adhesive restoration with fiber-reinforced plastic posts (FRP posts) in combination with composite resins that are specifically designed for core build-ups ${ }^{1-4)}$.

The clinical use of FRP posts has increased continuously since these materials were introduced in 1990 by Duret et al. ${ }^{5}$. FRP posts are composed of fibers of silica surrounded by a matrix of polymer resin, which is usually an epoxy resin ${ }^{6)}$. Therefore, the term 'fiber-reinforced plastic' (FRP) seems to be more suitable than fiber-reinforced composite (FRC), although the latter has frequently been used in the past ${ }^{4}$. FRP posts have an elasticity modulus very similar to that of dentin, and this may lead to a better distribution of the occlusal loads along the root $^{7-9)}$. Moreover, FRP posts are translucent, and therefore have distinct esthetic advantages ${ }^{6}$. With this host of advantages, FRP posts have been suggested recently as possible alternatives to cast metal post and core restorations of endodontically treated teeth ${ }^{10)}$.

Primarily, composite materials were not specifi- cally developed for core build-up procedures in combination with FRP posts. However, as a consequence of their properties, there have been indications for composite resins to be used as core materials ${ }^{11)}$. According to the manufacturers' recommendations, composite resin core build-up materials should be used with the respective adhesive systems to facilitate retention to the remaining dentin of the destroyed tooth ${ }^{2,12}$. The retention and stability of post and core systems are important factors for the success of a definitive restoration. Therefore, core build-up materials should exhibit good adaptation and reliable bond strength to the post surface ${ }^{11,13)}$.

The insertion of FRP posts requires separate conditioning of the intraradicular dentin with total-etch, self-etch bonding materials or self-adhesive resin cement systems ${ }^{14,15)}$. Following which, the composite resin core build-up material is usually placed on the remaining dentin in combination with the respective adhesive system in the cavity and around the FRP post ${ }^{12)}$.

Various composite materials are available, and their adhesion to FRP posts was the objective of several previous investigations ${ }^{4,11,16,17)}$. Typically, it is recommended by manufacturers to use composite resin core build-up materials in combination with their respective adhesive systems. However, to date, the influence on adaptation and bond strength of viscous composite resins to FRP posts has not been investigated and elucidated.

During the core build-up treatment procedure, it 
is an uphill - if not impossible - task for dental practitioners to condition the dentin without simultaneous application of the liquid components on the inserted FRP post surface. It should be noted that currently available FRP posts have highly crosslinked matrices. With the high conversion rate of epoxy resin, it can be assumed that the matrix is less reactive if there were no functional group available for reaction and bonding between FRP post and adhesive luting agent might be adversely affected $^{4)}$. With micromechanical retention playing a predominant role, bonding procedures without the use of respective adhesives may well be a feasible and favorable option.

Consequently, the objective of the present study was to evaluate the effect of post surface conditioning with adhesive systems on the tensile bond strength between two different types of composite resin cores and FRP posts. The null hypothesis of the study was that neither the type of composite resin core nor the post surface conditioning with the respective adhesive system has a significant influence on the tensile bond strength between FRP posts and composite abutments.

\section{MATERIALS AND METHODS}

FRP posts

Forty-eight fiber posts (ER DentinPost, Komet, Lemgo, Germany) of size 3 with a length of $20.0 \mathrm{~mm}$ and a maximum diameter of $2.0 \mathrm{~mm}$ were used for bond strength testing using a pull-out design. The ER DentinPost was a FRP post composed of unidirectional glass fibers $(65.0 \%$ weight, embedded in an epoxy resin matrix) with a gradient angle of $4.2^{\circ}$ (taper approximately 9\%) in the apical area and medium third $(12 \mathrm{~mm})$. The coronal third $(8 \mathrm{~mm})$ of the post was parallel.

The posts were trimmed at the coronal part to a length of $15 \mathrm{~mm}$. On the supposition that the posts might be inserted to a maximum length of $12 \mathrm{~mm}$ into the root canal, the specimens were obtained by covering the upper part $(3 \mathrm{~mm})$ of the posts with a standardized composite resin core build-up. Calculated bonding surface was $21.99 \mathrm{~mm}^{2}(2 \pi \mathrm{rh}+$ $\pi r^{2}=2 \times 3.14159 \times 1 \mathrm{~mm} \times 3 \mathrm{~mm}+3.14159 \times$ $\left.1 \mathrm{~mm}^{2}=21.99 \mathrm{~mm}^{2}\right)$.

Composite resin core build-ups

ClearfilCore (Kuraray, Okayama, Japan) and MultiCore Flow (Ivoclar Vivadent, Schaan, Liechtenstein) were used as core build-up materials

Table 1 Contents of the resin core build-up systems (information as provided by the manufacturers)

\begin{tabular}{|c|c|c|c|c|}
\hline & ClearfilCore & $\begin{array}{l}\text { Clearfil New Bond } \\
\text { (bonding system) }\end{array}$ & MultiCore Flow & $\begin{array}{l}\text { AdheSE } \\
\text { (bonding system) }\end{array}$ \\
\hline \multirow[t]{2}{*}{ Composition } & $\begin{array}{l}\text { Universal Paste } \\
\text { silanized silica, } \\
\text { TEGDMA, Bis-GMA, } \\
\text { N,N-diethanol-p- } \\
\text { toluidine }\end{array}$ & $\begin{array}{l}\text { Universal Paste } \\
\text { N,N-diethanol-p- } \\
\text { toluidine, sodium } \\
\text { benzensulfinate, } \\
\text { ethanol }\end{array}$ & $\begin{array}{l}\text { Bis-GMA, UDMA, } \\
\text { TEGDMA, barium } \\
\text { glass, ytterbium } \\
\text { trifluoride, Ba-Al- } \\
\text { fluorosilicate, silicon } \\
\text { dioxide, catalysts, } \\
\text { pigments, stabilizers }\end{array}$ & $\begin{array}{l}\text { Primer } \\
\text { Phosphonic acid } \\
\text { acrylate, BIS-acryl } \\
\text { acid amide, water, } \\
\text { initiators, stabilizers }\end{array}$ \\
\hline & $\begin{array}{l}\text { Catalyst Paste } \\
\text { silanized silica, } \\
\text { colloidal silica, } \\
\text { TEGDMA, Bis-GMA, } \\
\text { benzoyl peroxide }\end{array}$ & $\begin{array}{l}\text { Catalyst Liquid } \\
\text { Bis-GMA, MDP, } \\
\text { HEMA, DMA, } \\
\text { benzoyl peroxide }\end{array}$ & & $\begin{array}{l}\text { Bonding } \\
\text { DMA, HEMA, silicon } \\
\text { dioxide, initiators, } \\
\text { stabilizers }\end{array}$ \\
\hline Polymerization & Self-curing & Self-curing & Light curing, $30 \mathrm{~s}$ & Light curing, $30 \mathrm{~s}$ \\
\hline Manufacturer & \multicolumn{2}{|c|}{ Kuraray, Okayama, Japan } & \multicolumn{2}{|c|}{ Ivoclar Vivadent, Schaan, Liechtenstein } \\
\hline
\end{tabular}

TEGDMA (triethylene glycol dimethacrylate), Bis-GMA (bisphenol-a-diglycidylmethacrylate), UDMA (urethane dimethacrylate), MDP (10-methacryloyloxydecyl dihydrogen phosphate), HEMA (2-hydroxyethyl methacrylate), DMA (dimethacrylate) 
(Table 1). Prior to the application of each composite resin core build-up material, the bonding surfaces of half of the posts (24 posts) were treated with the respective bonding systems (Clearfil New Bond, Kuraray or AdheSE, Ivoclar Vivadent) of the composite materials. Four experimental groups were formed thereof:

Group 1: ER DentinPost and ClearfilCore $(\mathrm{n}=12)$;

Group 2: ER DentinPost and Clearfil New Bond/ ClearfilCore $(\mathrm{n}=12)$;

Group 3: ER DentinPost and MultiCore Flow $(\mathrm{n}=12)$;

Group 4: ER DentinPost and AdheSE/MultiCore Flow $(\mathrm{n}=12)$.

To prepare the core build-ups, a special apparatus to position the post in the center with reference to the composite build-up - was constructed. After each post was positioned upright in the device, the upper part of the apparatus - a removable nylon pattern with a standardized mold - was placed on the device. The mold fabricated core build-ups with a total abutment height of $9.5 \mathrm{~mm}$ and with two

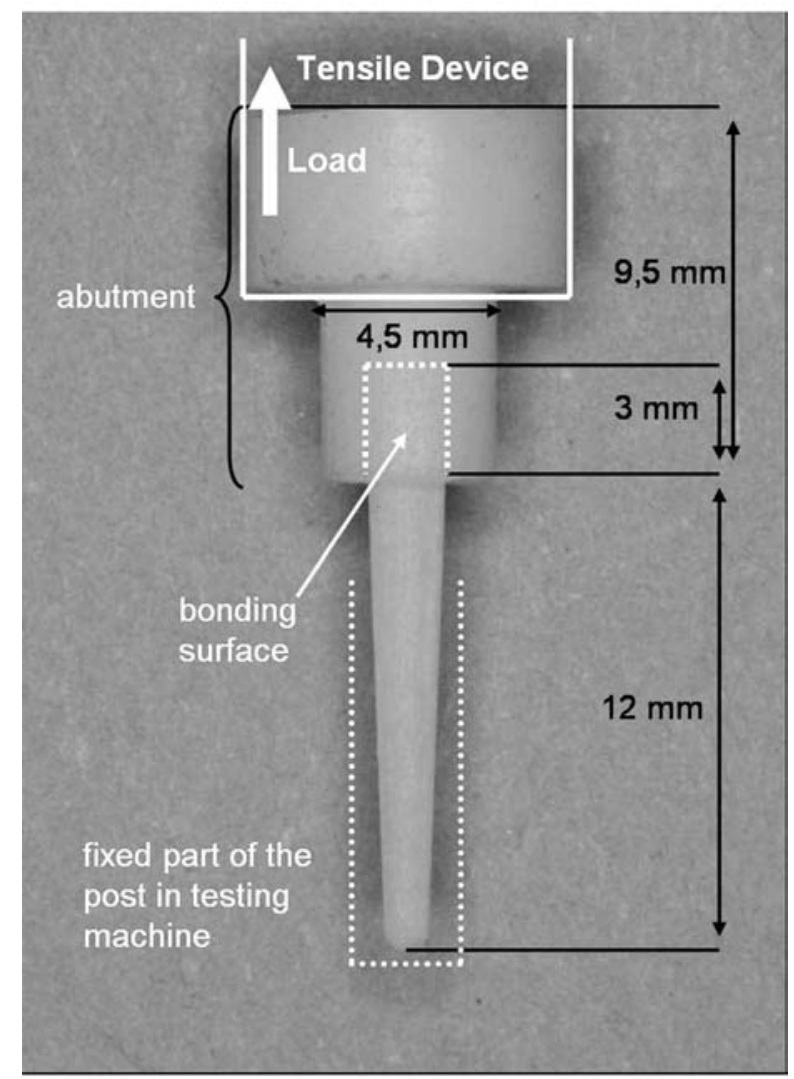

Fig. 1 Schematic illustration of experimental set-up (ER DentinPost with MultiCore Flow build-up). different diameters (diameter $=4.5 \mathrm{~mm}$ around the post; diameter $=8.7 \mathrm{~mm}$ in the upper part) (Fig. 1). The mold in the nylon pattern ensured a standardized shape of the composite core build-ups and equal distribution of the core material around the posts. Upper part of the abutment served as a retention interface for the tensile device of the universal testing machine (Instron Type 4204, Instron, Canton, MA, USA).

Adhesive systems and flowable composite core build-up materials were used according to manufacturers' recommendations for use on the dentin surface, as well as applied into the mold and around the upper $3 \mathrm{~mm}$ of the fiber posts.

1. Clearfil New Bond/ClearfilCore build-up procedure (Group 2)

Post surfaces were etched with $40 \%$ phosphoric acid gel (K-etchant gel, Kuraray) for 30 seconds, rinsed with a spray for at least 20 seconds, and carefully dried. Subsequently, the bonding surfaces were treated with a silane coupling agent (1\% in weight), Monobond-S (Ivoclar Vivadent), for 60 seconds using a disposable brush. After drying the post surfaces, the two components of Clearfil New Bond (Universal Liquid and Catalyst Liquid) were mixed in equal portions of $1: 1$, and a thin coat of the mixture was applied on the post surfaces. Mixing of the two pastes of the self-curing ClearfilCore followed, and the material was dispensed from a syringe (Dentsply, Konstanz, Germany).

2. AdheSE/MultiCore Flow build-up procedure (Group 4)

The separate etching procedure was administered with the self-etching adhesive (AdheSE primer). The primer was applied on the post surfaces, and the time allowed for reaction was 30 seconds. Excess amount of primer was dispersed with an oil-free stream of air, and the bonding surfaces were treated with Monobond-S for 60 seconds and dried. Then, AdheSE bonding was applied, carefully dispersed, and polymerized for 10 seconds using a halogen light (Optilux 401, Kerr, Demetron, Orange, CA, USA). MultiCore Flow was a dual-activated composite resin and dispensed directly from a syringe (MultiCore Refill Dispenser, Ivoclar Vivadent) in increments of 2 $\mathrm{mm}$ thickness, which were cured for 30 seconds using a halogen light (Optilux 401).

The composite resin was directly polymerized from the upper side of the mold. After removing the cured core build-up cylinder from the mold of the nylon pattern, a further irradiation of 60 seconds of the MultiCore Flow specimen was carried out from all sides in order to guarantee complete polymerization of the dual-curing composite resin core build-up material.

3. ClearfilCore and MultiCore Flow build-up procedure (Groups 1 and 3) 
In Groups 1 and 3, the respective adhesive systems of ClearfilCore and MultiCore Flow were not used. Silanization of the FRP posts was performed without application of K-etchant gel and Clearfil New Bond in Group 1 or AdheSE primer and AdheSE bonding in Group 3. The composite resin core build-up materials were applied after silanization of the untreated bonding surfaces with Monobond-S for 60 seconds.

Tensile bond strength test

After a storage period of seven days in a humid atmosphere $\left(100 \%\right.$ relative humidity at $\left.37^{\circ} \mathrm{C}\right)$, the specimens were mounted in a universal testing machine (Instron Type 4204), and the core composite resin abutments were fixed in the tensile testing device. The specimens were loaded at a crosshead speed of $1.0 \mathrm{~mm}$ per minute in a pull-out direction (Fig. 1) until the composite core build-ups were separated from the posts. Bond strength was expressed in MegaPascals ( $\mathrm{MPa}$ ) by dividing the load at failure (Newton) by the bonding surface $\left(\mathrm{mm}^{2}\right.$; see above).

\section{SEM analysis}

After tensile testing, the bonding surfaces were analyzed using a light microscope (Axioskop 40, Carl Zeiss, Jena, Germany) at $\times 36$ magnification. Following which, representative specimens were evaluated using scanning electron microscopy (SEM; LEO 435 VP, Oberkochen, Germany). Type of failure at the interface was determined as adhesive between post and composite resin core or cohesive within the composite resin core.

\section{Statistical analysis}

Results obtained from tensile testing were compared using two-way ANOVA and the Scheffé procedure (SPSS 12.0 for Windows, SPSS Inc., Chicago, Illinois, USA). Level of statistical significance was set at $\mathrm{p}<0.05$.

Table 2 Mean tensile bond strength values and standard deviations

\begin{tabular}{ccc}
\hline Group & n & Mean \pm SD $(\mathrm{MPa})$ \\
\hline ClearfilCore & 12 & $7.53 \pm 0.89^{\mathrm{A}}$ \\
Clearfil New Bond + ClearfilCore & 12 & $8.08 \pm 0.93^{\mathrm{A}}$ \\
MultiCore Flow & 12 & $5.80 \pm 0.39^{\mathrm{B}}$ \\
AdheSE + MultiCore Flow & 12 & $5.92 \pm 0.43^{\mathrm{B}}$ \\
\hline
\end{tabular}

Different superscript letters indicate significant differences $(p<0.05$, two-way ANOVA, Scheffé procedure)

\section{RESULTS}

Table 2 shows the bond strength values and standard deviations. Two-way ANOVA revealed that ClearfilCore achieved significantly higher bond strengths compared to MultiCore Flow $(p<0.0001)$.

The use of adhesive systems on the bonding surfaces of FRP posts increased the tensile bond strength. Indeed, mean bond strength values were higher when the adhesive systems were used, but these differences were not significant $(p>0.05$, twoway ANOVA).

ClearfilCore in combination with Clearfil New Bond resulted in a significantly higher value than MultiCore Flow with AdheSE $(\mathrm{p}<0.0001$, Scheffé procedure). Similarly, mean bond strength value was higher when FRP post surfaces were conditioned with a combination of phosphoric acid and Clearfil New Bond system (Group 2) versus the exclusive

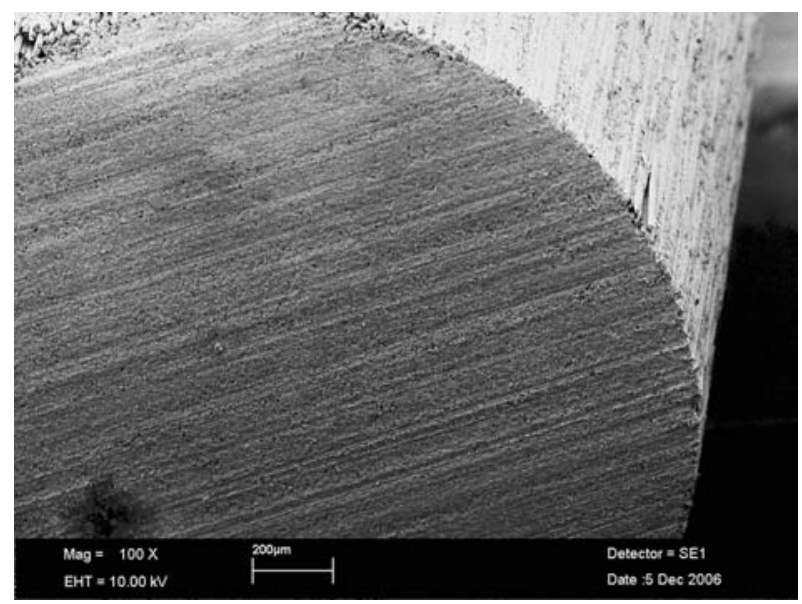

Fig. 2a SEM image $(\times 100)$ of a post sectioned from the coronal part to a length of $15 \mathrm{~mm}$ with a nontreated top surface. Fibers embedded in epoxy resin matrix were in the center and exposed at the margin of the post.

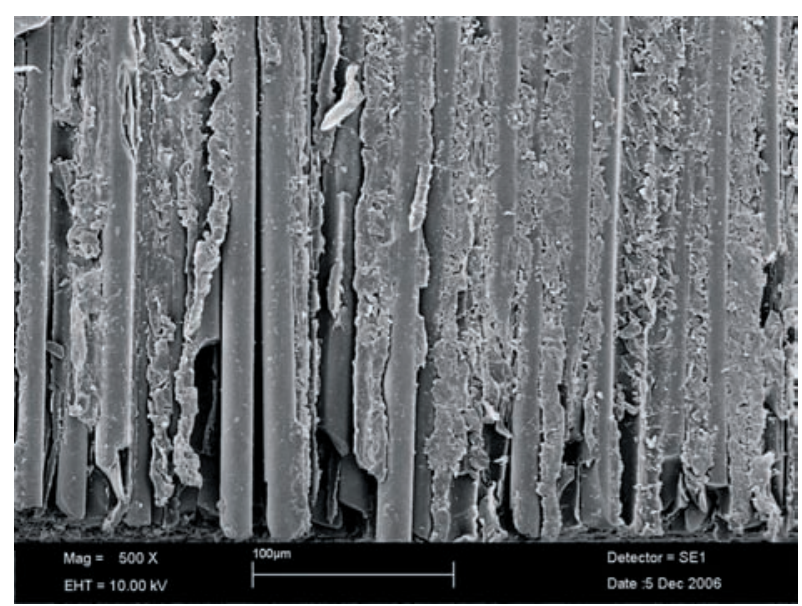

Fig. 2b SEM image $(\times 500)$ of non-treated side (axial surface). Fibers were exposed on the surface. 


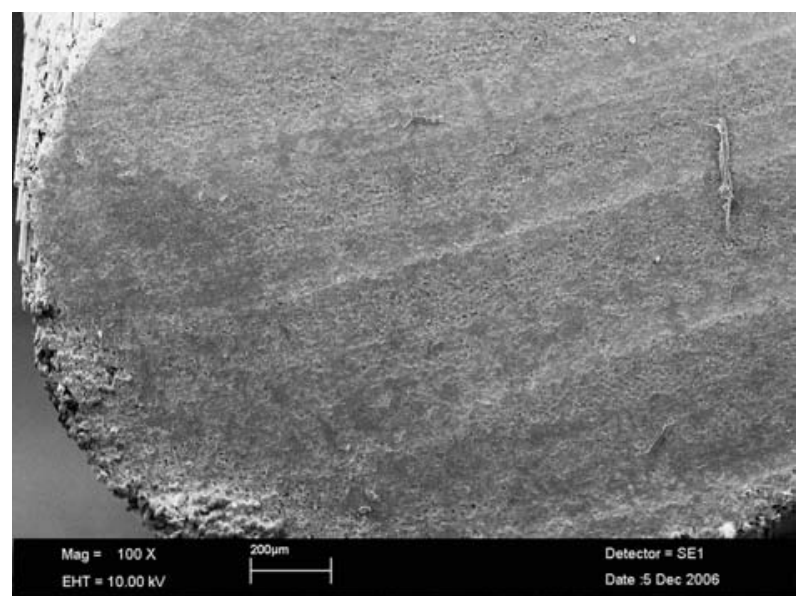

Fig. 3a SEM image $(\times 100)$ of a debonded and resincovered top surface (G2: Clearfil New Bond/ClearfilCore), demonstrating a cohesive failure mode within the adhesive resin.

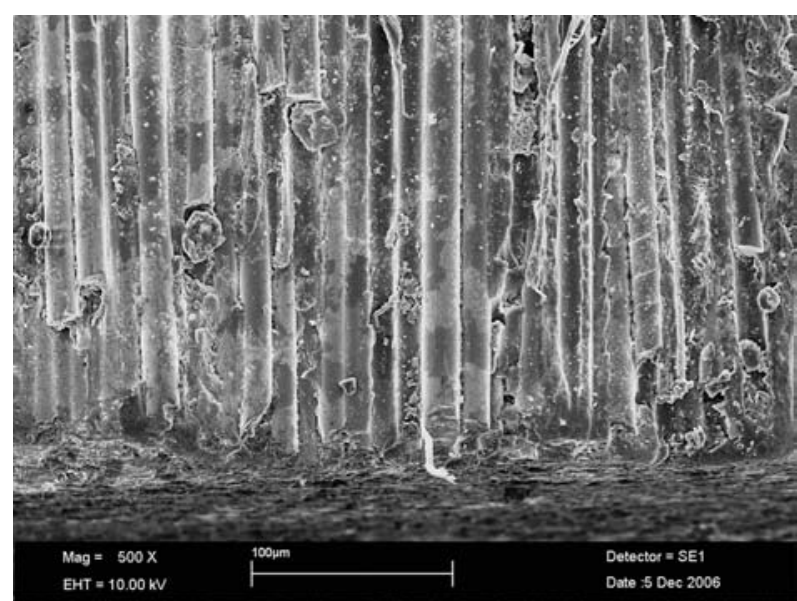

Fig. 3b SEM image $(\times 500)$ of a debonded side (axial surface) (G2: Clearfil New Bond/ClearfilCore). Pits and fissures between the glass fibers were filled with remnants of resin, indicating cohesive failure within the resin.

application of ClearfilCore (Group 1). However, Scheffé procedure revealed no significant differences ( $p>0.05)$. As with Groups 1 and 2, the difference between MultiCore Flow (Group 3) and MultiCore Flow with AdheSE (Group 4) was not significant ( $p>0.05$, Scheffé procedure).

Without exception, light microscopic evaluation of the bonding surfaces after the bond strength test showed that adhesive failure between the posts and core build-up materials occurred. SEM analysis of some representative specimens of Groups 2 and 4 demonstrated cohesive failure. As shown in Figs. 2 and 3, the SEM images showed a resin-covered top surface and remnants of resin on the lateral bonding surfaces of FRP posts.

\section{DISCUSSION}

In the present study, the adhesion of (flowable) composite resin core build-up materials with and without the use of respective bonding systems to FRP posts with unidirectional glass fibers $(65 \%$ weight, bound in an epoxy resin matrix) was assessed using a pull-out design. With this type of post - and hence with most commercially available fiber posts, epoxy resin is the matrix connecting the individual fibers ${ }^{4,18)}$. As for the storage duration of specimens before testing, it was based on studies which demonstrated that composite resins yielded the maximum bond strength after a storage period of seven days ${ }^{19}$.

Conventional methods for testing interfacial bond strength are namely the push-out and microtensile tests $^{4,9,18,20)}$. With the push-out test, fractures occur parallel to the bonding interface along the long axis of the posts, thus representing a shear bond test indeed ${ }^{21}$. As for the microtensile bond strength test, it is designed to test the interfacial bond strength between prefabricated FRP posts and composite resin cores. With this test method, serially sectioned 1mm-thick slices of post and core materials — with only a small cross-sectional area-are used as specimens $^{4,9,11,16)}$. The small bonded area between the resin and post is difficult to define exactly and has to be calculated because of the curvature of the interface ${ }^{9}$. Besides, the comparably high premature failure rate during specimen preparation is regarded as a glaring disadvantage of this method ${ }^{11}$.

As for the pull-out design used in the present study, it was a method that combined shear and tensile bond strength testing. In terms of specimen design, it entailed standardized abutments covering a well-defined bonding surface at the top of the post (tensile stress) as well as the long axis of the post (shear stress). In this manner, an improved simulation of the clinical situation was achieved - at least to some extent. Resulting bond strength values of this experiment then represented the total bond strength between the core build-up material and FRP post, as so defined in the bonding surface area for the composite resin core build-up.

In the present investigation, the maximum bond strength values were lower when compared to previous microtensile bond strength studies ${ }^{4,16}$. One reason might be the composition of the FRP post used. According to manufacturer's information, ER DentinPost consisted of $65.0 \%$ glass fibers in weight. This was somewhat higher than the previously used $^{4)}$ FRC Postec posts (Ivoclar Vivadent) which were composed of $61.5 \%$ (in weight) of glass. Based on these compositional data, the bonding surface of ER DentinPost thus had a lower content of epoxy resin matrix, which might reduce micromechanical and 
chemical bonding to the composite resin core. Thus, with regard to the percentage difference of fibers, the influence of exposed fibers at the abutting face of the posts could be considered as negligible.

According to manufacturer's information, MultiCore Flow had a filler content of $71 \%$ in weight, whereas ClearfilCore boasted a superior filler content of $78 \%$ in weight. Flowable composites presented good adaptation at the surface of FRP posts, but the mechanical properties of flowable composites resins are generally considered inferior if compared to conventional materials. Moreover, flowable composite resins have a lower filler-resin ratio than conventional composite resins ${ }^{17,22,23)}$. It has been reported that composite resins with higher filler content reveal lower polymerization shrinkage ${ }^{24-26)}$. On the other hand, a higher content of resin may lead to increased contraction during polymerization, whereby stress from shrinkage strain could weaken the interfacial bonds, thus affecting the bond strength to the post surface ${ }^{11}$.

Conversely, it has been demonstrated that highly filled packable composite resins were less capable of reducing contraction stress ${ }^{27}$. In the present study, the vectors of stress - arising from polymerization shrinkage - were directed toward the post. As such, it might be conceivable that increased contraction stress of the higher-filled ClearfilCore could have strengthened the interfacial bond between the post and core resin, thereby contributing to a form of shrinkage-related mechanical interlocking. Therefore, it could be said that differences in contraction stress between the two composite resins used might have additionally influenced the bond strength.

Polymerization shrinkage and contraction stress of dental resin composites depend on a variety of factors: unpolymerized resin content, type of resin monomer and composite system, setting mechanism, and curing mode ${ }^{28-32}$. In the present study, the difference in polymerization mode (i.e., self-curing versus dual photocuring mode) might have affected the outcome. As a result, the bond strength values of the highly filled ClearfilCore specimens were significantly higher than those of MultiCore Flow. An additional possible explanation for the higher bond strength values observed with ClearfilCore (compared to MultiCore Flow) might stem from the improved mechanical properties due to the higher filler content. Taken together, the higher bond strength and the resultant improved resistance to dislocation of ClearfilCore core build-ups were indicative of a stronger interfacial bond of the material to the FRP posts. However, the true reasons responsible for the observed outcome remained unclear. Therefore, with regard to the suggested explanations expressed above, further research to shed light on and illuminate this subject is clearly needed.

The use of silane coupling agents to improve the interfacial bond strength between composites and FRP posts has been a controversial and vigorously debated subject ${ }^{18)}$. A microtensile bond strength study demonstrated that the use of a silane agent significantly improved the bond strength between FRP posts and flowable composite core build-up materials ${ }^{4)}$. However, in a recent study, the bond strength of resin cements to FRP posts was not significantly affected by silanization used as a pretreatment $t^{33)}$. Indeed, there were indications that the purported effects of silanization appeared to be clinically negligible ${ }^{34)}$.

Different theories have been proposed and considered to explain the bonding mechanism of silane coupling agents in adhesive dentistry. However, the chemical reaction and bonding mechanism of silanes in the adhesion process is not yet completely understood ${ }^{4,35)}$. Nevertheless, none of the recent studies has revealed a negative effect caused by silanization of the bonding surfaces on the interfacial bond strength between FRP posts and composite materials. Instead, it has been generally assumed that silane improves the interfacial bond strength $^{4,34,36)}$. Hinging on this general consensus about the positive effect of silane coupling agents, the bonding surfaces of FRP posts in the present study were treated with Monobond-S prior to the build-up procedure. However, it should also be emphasized that investigation into the effects of silanization was not the aim of the present study.

Different types of conditioning treatments have been investigated for the post surface with a view to arriving at a qualified pretreatment procedure for the bonding surfaces of FRP posts ${ }^{4,9,36,37)}$. On this note, tribochemical silica coating has been reported to increase the bond strength between FRP posts and resin cement when compared to surface etching with phosphoric or hydrofluoric acid ${ }^{9}$. In the present study, the post surfaces were conditioned with the adhesive systems - which were used according to the manufacturers' recommendations for dentin treatment.

AdheSE was a two-step self-etching bonding system. According to manufacturer's information, AdheSE primer consisted of monomers with acidic phosphate groups. The conditioning of FRP surfaces was thus undertaken by these self-etching monomers. As for Clearfil New Bond, it required a preceding surface etching procedure with phosphoric acid gel. After etching with K-etchant gel, rinsing and drying of the post surfaces followed.

It is known that etching procedures affect the surface topography of FRP posts. However, the effects of phosphoric acid are negligible compared to the use of hydrofluoric $\mathrm{acid}^{9}$. Nevertheless, the 
application of K-etchant gel might have caused minimal modification of the bonding surface and contributed to a cleaning effect of the available bonding surface. This procedure might have thus intensified the silane layer and improved micromechanical interlocking, thereby resulting in a slightly higher bond strength value of Group 2 (Clearfil New Bond and ClearfilCore) as compared to Group 1. Unlike phosphoric acid etching, the layer of acidic monomers of self-etching AdheSE system was left on the post surface. Nevertheless, statistical analysis revealed that the observed enhancement was not significant. Instead, the results indicated only a slight improvement of the interfacial bond when AdheSE system was applied on the post surfaces.

As with previous investigations, light microscopic and SEM analyses of the bond surfaces demonstrated exclusively adhesive bond failure after testing and debonding. This was in agreement with the results of recent microtensile bond strength studies, whereby bond failures of composite-fiber post units revealed only adhesive failure ${ }^{4,9)}$.

It has been assumed that some free radicals of the matrix of fiber posts form a chemical bond with BIS-GMA-based resins ${ }^{12)}$. However, the adhesive failure mode of the present study indicated that there were no chemical bonds between the composite materials and fiber posts. Mannocci et $a l^{38)}$ proved that monomers of bonding resins penetrated into the interpenetrating polymer network (IPN) polymer structure of EverStick Post, whereas no penetration into the crosslinked matrix was observed for another FRP post system. In other words, not all the monomers used in bonding resins are capable of infiltrating PMMA-based IPN structures. It should be highlighted that in the present study, the matrix of ER DentinPost did not consist of any IPN structure.

Notwithstanding, SEM analysis of representative samples clearly revealed remnants of bonding layer. It could be speculated that the bonding between resin cement and FRP post was a frail one at the resinpost-interface. Hence, the observed failure mode could rather be described as cohesive failure inside the resin-adhesive interdiffusion zone. In the present study, only a limited number of samples were additionally evaluated using SEM, whereas the complete analysis of debonding failure was conducted using a light microscope. Results of SEM analysis clearly demanded for further studies in this field. This was because the commonly used failure mode descriptions at the adhesive interface between post and resin cement seemed to be questionable.

Based on the results of the present study, the resistance to tensile and shear bond forces of composite resins bonded to fiber posts seemed to be solely attributed to micromechanical interlocking and sliding friction. Low-viscosity monomers of the adhesive systems might have penetrated and infiltrated the space between the glass fibers and microporosities in the epoxy resin matrix. The polymerized bonding resins then contributed to stabilizing and enhancing the micromechanical retention between polymers and FRP posts. Thus, the null hypothesis - that neither the type of composite resin core nor the post surface conditioning with the respective adhesive system has any influence on the tensile bond strength between FRP posts and composite core build-ups — had to be partly rejected.

\section{CONCLUSIONS}

Within the limitations of this in vitro study, it could be concluded that the investigated pretreatment procedures had only a minor influence on bond strength. Therefore, pretreatment of fiber posts with the respective adhesive systems did not seem to be mandatory. Moreover, during the composite resin core build-up procedure, dentin conditioning could proceed with simultaneous application of the liquid components on the inserted FRP post - thereby saving time in the clinical situation.

Differences in bond strength observed with the various composite resins were significant. In other words, bond strength to FRP posts was dependant on the composite resin systems used. Nonetheless, with regard to long-term bonding, further studies after thermomechanical loading and water storage of the samples are clearly needed.

\section{REFERENCES}

1) Schwartz RS, Robbins JW. Post placement and restoration of endodontically treated teeth: a literature review. J Endod 2004; 30:289-301.

2) Combe EC, Shaglouf AMS, Watts DC, Wilson NHF. Mechanical properties of direct core build-up materials. Dent Mater 1999; 15:158-165.

3) Saygili G, Mahmali SM. Comparative study of the physical properties of core materials. Int $\mathrm{J}$ Periodont Rest Dent 2002; 22:355-363.

4) Goracci C, Raffaelli O, Monticelli F, Balleri B, Bertelli E, Ferrari M. The adhesion between prefabricated FRC posts and composite resin cores: microtensile bond strength with and without post-silanization. Dent Mater 2005; 21:437-444.

5) Duret B, Reynaud M, Duret F. New concept of coronoradicular reconstruction, the Composipost (1). Chir Dent Fr 1990; 60:131-141.

6) Bateman G, Ricketts DNJ, Saunders WP. Fibre-based post systems: a review. Br Dent J 2003; 195:43-48.

7) Galhano GA, Valandro LF, de Melo RM, Scotti R, Bottino MA. Evaluation of the flexural strength of carbon fiber-, quartz fiber-, and glass fiber-based posts. J Endod 2005; 31:209-211.

8) Lassila LVJ, Tanner J, Le Bell AM, Narva K, Vallitu 
PK. Flexural properties of fiber reinforced root canal posts. Dent Mater 2004; 20:29-36.

9) Valandro LF, Yoshiga S, de Melo RM, Galhano GÁP, Mallmann A, Marinho CP, Bottino MA. Microtensile bond strength between a quartz fiber post and a resin cement: effect of post surface conditioning. J Adhes Dent 2006; 8:105-111.

10) Monticelli F, Grandini S, Goracci C, Ferrari M. Clinical behavior of translucent fiber posts: a 2-year prospective study. Int J Prosthod 2003; 16:593-596.

11) Sadek FT, Monticelli F, Goracci C, Tay FR, Cardoso $\mathrm{PE}$, Ferrari M. Bond strength performance of different resin composites used as core materials around fiber posts. Dent Mater 2007; 23:95-99.

12) Ferrari M, Scotti R. Fiber posts characteristics and clinical applications, 1st ed, Masson, Milano, Italy, 2004, pp.25-120.

13) Gateau P, Sabek M, Dailey B. Fatigue testing and microscopic evaluation of post and core restorations under artificial crowns. J Prosthet Dent 1999; 82:341347.

14) Goracci C, Sadek FT, Fabianelli A, Tay FR, Ferrari M. Evaluation of the adhesion of fiber posts to intraradicular dentin. Oper Dent 2005; 30:627-635.

15) Wrbas KT, Kampe MT, Schirrmeister JF, Altenburger MJ, Hellwig E. Retention of fiber posts dependent on different resin cements. Schweiz Monatsschr Zahnmed 2006; 116:18-24.

16) Salameh Z, Papacchini F, Ounsi HF, Goracci C, Tashkandi E, Ferrari M. Adhesion between prefabricated fiber-reinforced posts and different composite resin cores: a microtensile bond strength evaluation. J Adhes Dent 2006; 8:113-117.

17) Monticelli F, Goracci C, Grandini S, Garcia-Godoy F, Ferrari M. Scanning electron microscopic evaluation of fiber post-resin core units built up with different resin composite materials. Am J Dent 2005; 18:61-65.

18) Perdigao J, Gomes G, Lee IK. The effect of silane on the bond strengths of fiber posts. Dent Mater 2006; 22:752-758.

19) Braga RR, Ballester RY, Carrilho MRO. Pilot study on the early shear strength of porcelain-dentin bonding using dual-cure cements. J Prosthet Dent 1999; 81:285-289.

20) Goracci C, Tavares AU, Fabianelli A, Monticelli F, Raffaelli O, Cardoso PC, et al. The adhesion between fiber posts and root canal walls: comparison between microtensile and push-out bond strength measurements. Eur J Oral Sci 2004; 112:353-361.

21) Drummond JL, Sakaguchi RL, Racean DC, Wozny J, Steinberg AD. Testing mode and surface treatment effects on dentin boning. J Biomed Mater Res 1996; $32: 553-541$

22) Labella R, Lambrechts P, Van Meerbeck B, Vanherle G. Polymerization shrinkage and elasticity of flowable composites and filled adhesives. Dent Mater 1999; 15:128-137.

23) Monticelli F, Goracci C, Ferrari M. Micromorphology of the fiber post-resin core unit: a scan ning electron microscopy evaluation. Dent Mater 2004; 20:176-183.

24) Alvarez-Gayosso C, Barcelo-Santana F, GuerreroIbarra J, Saez-Espinola G, Canseco-Martinez MA. Calculation of contraction rates due to shrinkage in light-cured composites. Dent Mater 2004; 20:228-235.

25) Chung KH, Greener EH. Correlation between degree of conversion, filler concentration and mechanical properties of posterior composite resins. J Oral Rehabil 1990; 17:487-494.

26) Davidson CL, Feilzer AJ. Polymerization shrinkage and polymerization shrinkage stress in polymer-based restoratives. J Dent 1997; 25:435-440.

27) Chen HY, Manhart J, Hickel R, Kunzelmann KH. Polymerization contraction stress in light-cured packable composite resins. Dent Mater 2001; 17:253259.

28) Kleverlaan CJ, Feilzer AJ. Polymerization shrinkage and contraction stress of dental resin composites. Dent Mater 2005; 21:1150-1157.

29) Atai M, Watts DC, Atai Z. Shrinkage strain-rates of dental resin-monomer and composite systems. Biomaterials 2005; 26:5015-5020.

30) Tachibana K, Kuroe T, Tanino Y, Satoh N, Ohata N, Sano H, Caputo AA. Effects of incremental curing: on contraction stresses associated with various resin composite buildups. Quintessence Int 2004; 35:299-306.

31) Kuroe T, Tachibana K, Tanino Y, Satoh N, Ohata N, Sano H, Inoue N, Caputo AA. Contraction stress of composite resin build-up procedures for pulpless molars. J Adhes Dent 2003; 5:71-77.

32) Feng L, Suh BI. The effect of curing modes on polymerization contraction stress of a dual cured composite. J Biomed Mater Res B Appl Biomater 2006; 76:196-202.

33) Bitter K, Meyer-Lueckel H, Priehn K, Martus P, Kielbassa AM. Bond strengths of resin cements to fiber-reinforced composite posts. Am J Dent 2006; 19:138-142.

34) Bitter K, Noetzel J, Neumann K, Kielbassa AM. Effect of silanization on bond strengths of fiber posts to various resin cements. Quintessence Int 2007; 38:121-128.

35) Matinlinna JP, Lassila LVJ, Özcan M, Yli-Urpo A, Vallittu PK. An introduction to silanes and their clinical application in dentistry. Int $\mathrm{J}$ Prosthodont 2004; 17:155-164.

36) Cheleux N, Degrange M, Sharrock P. Effets des différents conditionnements de surface sur l'adhésion du tenon light-post $\mathbb{R}^{\mathbb{P}}$. Eur Cell Mater 2005; 9:56-57.

37) Sahafi A, Peutzfeldt A, Asmussen E, Gotfredsen K. Bond strength of resin cement to dentin and to surface-treated posts of titanium alloy, glass fiber, and zirconia. J Adhes Dent 2003; 5:153-162.

38) Mannocci F, Sherriff M, Watson TF, Vallitu PK. Penetration of bonding resins into fibre-reinforced composite posts: a confocal micrsoscopic study. Int Endod J 2005; 38:46-51. 